\begin{tabular}{|l|l|c|c|}
\hline Eiszeitalter $u$. Gegenwart & 39 & $19-28$ & Hannover 1989 \\
\hline
\end{tabular}

\title{
Schwermineralanalyse von Geschiebemergeln, ein Hilfsmittel für die Moränenstratigraphie? Diskussion anhand von Zählungen aus Schleswig-Holstein
}

\author{
Uwe Hentschke \& Hans-Jürgen Stephan *)
}

\author{
Critical review, till, stratigraphy, methods, \\ heavy minerals, modal analysis, mineral assemblages, spatial distribution \\ Schleswig-Holstein
}

\begin{abstract}
Kurzfassung: Aus der Literatur entnommene Schwermineralanalysen von Geschiebemergeln werden mit einigen neuen Analysen zusammen ausgewertet. Von den elsterzeitlichen bis zu den weichselzeitlichen Geschiebemergeln gibt es in den Schwermineralassoziationen zwar deutliche Verschiebungen, jedoch zeigen die Auswertediagramme breite, sich meist stark überlappende Streufelder. Eine eindeutige Unterscheidung der Geschiebemergel aus aufeinanderfolgenden Vereisungen ist nicht möglich. Die Schwermineralassoziation kennzeichnet - wie auch andere petrographische Parameter - eine bestimmte Grundmoränenfazies. Sie kann sich in gleich alten Moränen regional ändern, aber auch in verschieden alten Moränen in ähnlicher Zusammensetzung auftreten.
\end{abstract}

[Heavy Mineral Analysis of Tills, a Method for Till Stratigraphy?

A Discussion on the Basis of Counts from Schleswig-Holstein]

\begin{abstract}
Heavy mineral analyses of tills from SchleswigHolstein published by several authors are evaluated together with some new analyses. From Elsterian to Weichselian tills the heavy mineral associations show distinct trends, but broad and in most cases strongly overlapping dispersion fields are found in the evaluation diagrams. A clear distinction of tills of successive glaciations is not possible. As other petrographical parameters also the heavy mineral association indicates a specific ground moraine facies. It can both change regionally in coeval moraines and be found with a similar composition in moraines of different age.
\end{abstract}

*) Anschriften der Verfasser: Dr. U. Hentschke, Physikzentrum der Christian Albrechts Universität, Institut für Angewandte Physik, Olshausenstr. 40-60, 2300 Kiel 1; Dr. H.-J. Stephan, Geologisches Landesamt Schleswig-Holstein, Mercatorstr. 7, 2300 Kiel 1.

\section{Einleitung}

Obwohl die Methode, glaziale Ablagerungen durch ihre Schwermineralgemeinschaft zu charakterisieren und zu korrelieren, schon seit langem erprobt und in verschiedenen Gebieten offenbar mit Erfolg angewandt wurde (u. a. RAISTRICK 1929; LEINZ 1933; PRATJE \& RiCHTER 1938), hat in Schleswig-Holstein nach ersten, noch unvollkommenen Versuchen von FIEDLER (1939) in größerem Umfang erst STEINERT (1952) die Schwermineralvergesellschaftungen glazialer Ablagerungen mit dieser Zielrichtung untersucht. Er bestätigte dabei einige wesentliche, schon von FIEDLER erkannte Grundzüge: Als generelle Gesetzmäßigkeiten fand er, daß Amphibole und Pyroxene in den jüngeren Ablagerungen relativ angereichert sind, dagegen opake Schwerminerale dort die niedrigsten Werte erreichen. In älteren Ablagerungen nimmt der Gehalt an Amphibolen und Pyroxenen kontinuierlich ab und opake Minerale, Minerale aus metamorphen Gesteinen sowie Epidot treten dementsprechend häufiger auf. STEINERT erklärte diese Tendenzen damit, daß das Inlandeis der jüngeren Vereisungen u. a. in größerer Menge frische Hornblenden aus den Kristallingebieten Skandinaviens mitgebracht hätte, die Gletscher der älteren Vereisungen dagegen noch stärker der Ausschürfung zugängliche tertiäre Sedimente mit ihrer Vormacht stabiler Schwerminerale aufgearbeitet hätten. Er schloß aus seinen Untersuchungen, daß eine schwermineralanalytische Gliederung des schleswig-holsteinischen Pleistozäns, insbesondere die Abtrennung weichselzeitlicher von älteren Ablagerungen, möglich sei (1952: 109). Als Richtwert gab er an, daß das Verhältnis von Hornblende zu Horn- 
blende + Epidot in weichselzeitlichen Ablagerungen $\geqq 0,7$ („Hornblendezahl 70 ”) sei, in älteren kleiner. Pieles (1955), Hölting (1957) und WeISS (1958) versuchten, mit Hilfe der STEINERT'schen Vorgaben in verschiedenen Gebieten Schleswig-Holsteins weichselzeitliche von saalezeitlichen Sedimenten zu trennen. In erster Linie wurden Schmelzwasserablagerungen untersucht. Während WeISS im Bezirk Schleswig und HÖLTING im nördlichen und mittleren Holstein die STEINERT'sche Richtzahl im wesentlichen mit Erfolg nutzen konnten, stellte PIELES im südöstlichen Schleswig-Holstein abweichende Verhältnisse fest, die den Erwartungen widersprachen. Ergänzungshalber soll noch eine Arbeit von BIERMANN (1981) erwähnt werden, die eine Teilanalyse der SM-Zusammensetzung der weichselzeitlichen Moräne von Timmerhorn durchführte.

Im Rahmen einer Arbeit, die schwerpunktmäßig die plio-pleistozänen Ablagerungen Niedersachsens betraf, analysierte SCHLENKER auch die Schwermineralassoziationen mehrerer Geschiebemergel aus Schleswig-Holstein (MEYER \& SCHLENKER 1979). Er bekräftigte die wesentlichen Erkenntnisse von STEINERT. Weitere seiner Zählergebnisse aus Geschiebemergeln bei Hemmoor sind in HÖFLE (1979) und HÖFLE \& SCHLENKER (1979) publiziert. Mit den Schwermineralen in glazialen Sedimenten Niedersachsens befassen sich ebenfalls SCHRÖDER (in DUPHORN 1983: 78) und HENNINGSEN (1987). Nach Ansicht von HENNINGSEN können Granat und Augit ins Verhältnis gesetzt zu den ausgeprägt Metamorphitmineralen Disthen, Staurolith und Sillimanit („Granatzahl” und „Augitzahl"), besonders in der Summe, am besten bei der altersmäßigen Abgrenzung von glazialen Sedimentserien helfen. Die Masse der von ihm benutzten Daten lieferten jedoch Zählungen von sandigen Sedimenten unterschiedlicher Genese.

SCHRÖDER kommt im wesentlichen zum Ergebnis, daß die Zusammensetzung von Schwermineralgemeinschaften in den glazialen Sedimenten NE-Niedersachsens stark schwankt und daß sich dort SM-Zählungen für die Trennung saalezeitlicher Geschiebemergel von elsterzeitlichen und der verschiedenen saalezeitlichen untereinander nicht eignen.

Für Schleswig-Holstein hat es nach der Publikation von STEINERT keine größere Untersuchung über die Schwermineralführung von im engeren Sinn glaziären Ablagerungen, der Geschiebemergel, gegeben. Für die Klärung der Stratigraphie von Geschiebemergeln (Grundmoränen) wurden andere petrographische Methoden eingesetzt (s. Auflistung bei STEPHAN 1987: 24).

\section{Neue Schwermineralanalysen an Geschiebemergeln}

Trotz einiger Erfolge bei der stratigraphischen Zuordnung von Geschiebemergeln mit Hilfe verschiedener petrographischer Untersuchungen gab es auch immer wieder Schwierigkeiten und Fehlschläge. So war es z. B. bisher nicht möglich, die petrographisch gut trennbaren Geschiebemergel des „Hohen Ufers” von Heiligenhafen - mit Ausnahme des jüngsten sicher einer bestimmten Eiszeit zuzuordnen (STEPHAN 1985). Die Schwermineralanalyse bot sich hier als weitere noch nicht genutzte Untersuchungsmethode an.

Um die aus der Literatur bekannten Analysenergebnisse über die SM-Zusammensetzung von Geschiebemergeln zu ergänzen, wurden 21 Proben aus allen zur Zeit altersmäßig unterschiedenen Geschiebemergeln aus verschiedenen Teilen Schleswig-Holsteins ausgewählt.

\subsection{Aufbereitung und Methodik}

Der Großteil der Proben wurde aus der bei der Gewinnung von Feinkiesproben für die Feinkiesanalyse anfallenden Sandfraktion abgesiebt, danach über einen Zentrifugen-Probenteiler mehrfach geteilt.

In der Regel reichen $3 \mathrm{~g}$ Probenmaterial für eine anschließende Schwerkraftabtrennung mittels Dichtelösung aus.

Das Probenmaterial wurde im Ultraschallbad auf ein $60 \mu \mathrm{m}$-Sieb gegeben. Nach vollständiger Abtrennung der Feinfraktion wurde der restliche Anteil bei $100^{\circ} \mathrm{C}$ getrocknet. Anschließend wurde die Fraktion über $0,25 \mathrm{~mm}$ abgesiebt und verworfen. Auch die Feinfraktion wurde nicht weiter bearbeitet und verworfen, da eine Schwerkraftabtrennung der Körner mit diesen kleinen Durchmessern in einer Dichtelösung zu ungenau ist (BOENIGK 1983: 12); außerdem ist ihre optische Bestimmung im Präparat kritisch. Als Dichtelösung wurde abweichend von der in früheren Arbeiten publizierten Methodik Natriumpolywolframat benutzt. Diese Lösung ist im Chemikalienhandel erhältlich und hat den Vorteil, daß sie wasserlöslich und nach Angabe des Herstellers ungiftig ist. Damit kann sie auch in Laborräumen ohne Abzug verwendet werden.

Als Nachteil zeigte sich, daß diese Lösung bei Dichten um ca. 2.9 ausgesprochen niedrigviskos ist und im Vergleich zu den üblichen Dichtelösungen wie Bromoform oder Tetrabromäthan schlecht abfiltriert werden kann.

Von dem abgesiebten Material wurden ca. $2 \mathrm{~g}$ in einen $250 \mathrm{ml}$ Scheidetrichter mit Dichtelösung gegeben und 
Tab. 1: Auflistung der untersuchten Proben

\begin{tabular}{|c|c|c|c|c|c|}
\hline Nr. & Ort & Lage $\mathrm{R} / \mathrm{H}$ & Tiefe (m) & Zeitliche Einstufung & Beschreibung; Bemerkungen \\
\hline 1 & St. Michaelisdonn & $3507600 / 5985360$ & 8 & ältere Saalemoräne (qs AG, Burg-Formation) & Normalfazies; Aufschluß \\
\hline 2 & $\mathrm{HH}-$ Niendorf & $3565000 / 5943600$ & & jüngere Saalemoräne (qs JG, Fuhlsbüttler Moräne) & \\
\hline 3 & Besdorf & $3524530 / 5990035$ & 9 & mittlere Saalemoräne (qs MG, Kuden-Formation) & Bohrung Besdorf-Ost 6 \\
\hline 4 & Owschlag & $3539420 / 6026770$ & $48-49$ & Saalemoräne (qs) & \\
\hline 5 & Groß Niendorf & $3577875 / 5965350$ & 18 & jüngere Saalemoräne (qs JG) & \\
\hline 6 & Wacken & $3526160 / 5928255$ & & ältere Saalemoräne (qs AG, Burg-Formation) & Normalfazies; Neue Tongrube \\
\hline 7 & Kuden & $3513025 / 5982800$ & 9 & ältere Saalemoräne (qs AG, Burg-Formation) & $\begin{array}{l}\text { rotbrauner Geschiebemergel, ost- } \\
\text { baltische Fazies; Aufschluß Nord III }\end{array}$ \\
\hline 8 & Kuden & $3512690 / 5982075$ & 11 & ältere Saalemoräne (qs AG, Burg-Formation) & $\begin{array}{l}\text { Normalfazies; „unterer” Geschiebe- } \\
\text { mergel, Aufschluß Nord IV }\end{array}$ \\
\hline 9 & Hamwohrberg & $3567233 / 5988780$ & 18,5 & jüngere Saalemoräne (qs JG) & \\
\hline 10 & Eggstedterholz & $3517542 / 5993450$ & $168,8-168,9$ & Elstermoräne (qe) & schwärzlich \\
\hline 11 & Böxlund & $3511325 / 6078585$ & 7 & jüngere Saalemoräne (qs JG) & nördliche Fazies \\
\hline 12 & Wapelfeld & $3540240 / 5994175$ & 5 & mittlere Saalemoräne (qs MG) & \\
\hline 13 & $\begin{array}{l}\text { Lauenburg } \\
\text { Kuhgrund }\end{array}$ & $4402005 / 5916675$ & 5 & ?jüngere Saalemoräne (qs JG) & $\begin{array}{l}\text { bisher als qsMG eingestuft; nach } \\
\text { FK- u. chem. Analysen aber } \\
\text { vermutlich jünger }\end{array}$ \\
\hline 14 & Brüggerholz & $3571300 / 6007300$ & 7 & $\begin{array}{l}\text { Weichselmoräne, 1. Vorstoß } \\
\text { (qw, Brügge-Formation) }\end{array}$ & Aufschluß \\
\hline 15 & Brüggerholz & $3571400 / 6007225$ & 9 & jüngere Saalemoräne (qs JG) & Aufschluß \\
\hline 16 & Stubbenberg & $3515325 / 5983170$ & 9 & ältere Saalemoräne (qs AG, Burg-Formation) & $\begin{array}{l}\text { Mischfazies; braun, z. T. rotstichig; } \\
\text { Aufschluß II }\end{array}$ \\
\hline 17 & Holtenau & $3573725 / 6027745$ & 2,5 & $\begin{array}{l}\text { Weichselmoräne, jüngster Vorstoß } \\
\text { (qw, Fehmarn-Formation) }\end{array}$ & Aufschluß; „jungbaltische” Moräne \\
\hline 18 & Ratzeburg & $4419235 / 5952307$ & $172-174$ & Elstermoräne (qe) & \\
\hline 19 & Prehnsfelde & $3559287 / 5996890$ & $173-176$ & Elstermoräne (qe) & untere Elstermoräne \\
\hline 20 & Brodtener Ufer & $4426107 / 5984897$ & 4 & $\begin{array}{l}\text { Weichselmoräne, jüngster Vorstoß } \\
\text { (qw, Fehmarn-Formation) }\end{array}$ & ,jungbaltische” Moräne \\
\hline 21 & BAB Lägerdorf & $3524530 / 5990035$ & 3 & mittlere Saalemoräne (qs MG, Vaale-Formation) & \\
\hline
\end{tabular}


aufgeschüttelt. Nach ca. 1-2 Stunden konnte die abgesetzte Schwermineralfraktion abgetrennt werden. Dieser Vorgang wurde drei- bis viermal wiederholt, um die Schwerminerale wirklich quantitativ abzutrennen.

Die gewonnene Schwermineralfraktion wurde anschließend bei $60^{\circ} \mathrm{C}$ getrocknet und dann auf einem Objektträger in Kanadabalsam eingebettet. Hierbei wurde darauf geachtet, daß das Streupräparat nicht zu dicht belegt war. Die eventuell überschüssige SMFraktion wurde auf einem zweiten Objektträger eingebettet.

In den Präparaten wurden je Probe 500 Mineralkörner mit dem Polarisationsmikroskop bestimmmt und ausgezählt. Die Abweichungen bei Mehrfachzählungen sind vernachlässigbar, daher ist in den Tabellen jeweils nur eine Zählung angegeben.

In feinkornreichen Proben werden bei Kornzählungen die Verhältnisse stark zugunsten der bevorzugt feinkörnig auftretenden Schwerminerale wie beispielsweise Zirkon verschoben und dadurch im Vergleich zu sonstigen Geschiebemergelproben mit dem üblicherweise ausgeprägt breiten Korngrößenspektrum stark verzerrt (vgl. Probe 8, Kuden Nord IV; Tab. 2). In den Präparaten vom Hohen Ufer Heiligenhafen wurden daher vergleichsweise die Flächenprozente mit Hilfe eines Rasterokulars ausgezählt. Insgesamt wurden hierbei zwischen 1000 und 1200 Einzelpunkte bestimmt. Da dieses Verfahren aber nur selten angewendet wird und keine Vergleichswerte existieren, mußte für die stratigraphischen Auswertungen auf die sonst übliche Kornzählung zurückgegriffen werden. *)

\subsection{Diskussion der Analysenergebnisse}

Die Literaturdaten wurden sehr kritisch durchgesehen, und für die vergleichende Auswertung in den Diagrammen $1-7$ wurde auf die Werte aller derjenigen Proben verzichtet, die nach heutiger Kenntnis zeitlich nicht sicher einzuordnen sind. Außerdem konnte eine gewisse Anzahl der Analysen von STEINERT nicht verwendet werden, weil in seinen Tabellen offensichtliche Fehler vorkommen.

Aus Abb. 1 ergibt sich, daß - wie von FiedleR (1939, 1940) und von STEINERT (1952) festgestellt - weichselzeitliche Geschiebemergel hornblendereicher sind als ältere, es wird aber auch deutlich, daß es keine klare Trennung gibt. Die Linie für die STEINERT'sche Hornblendezahl 70 stellt keine Grenzlinie zwischen

*) Bei den Proben vom Hohen Ufer Heiligenhafen konnten Streupräparate der Fraktion 63-355 $\mu \mathrm{m}$ genutzt werden, die P. SCHRÖDER, vormals Geologisches Institut der Universität in Kiel, angefertigt hatte.



Abb. 1: Hornblende gegen Epidot + Zoisit in \% der durchscheinenden Schwerminerale

in Geschiebemergeln Schleswig-Holsteins.

$\mathrm{a}=$ weichselzeitlicher, $\mathrm{b}=$ roter weichselzeitlicher,

$c=$ jüngerer saalezeitlicher, $d=$ mittlerer saalezeitlicher, $\mathrm{e}=$ älterer saalezeitlicher, $\mathrm{f}=$ roter älterer saalezeitlicher, $\mathrm{g}=$ saalezeitlicher (ungegliedert),

$\mathrm{h}=$ elsterzeitlicher Geschiebemergel.

den Streufeldern weichselzeitlicher und älterer Geschiebemergel dar. So fallen die Werte aus dem sicher weichselzeitlichen „oberen Geschiebemergel” vom Heiligenhafener Kliff teilweise in das erwartete präweichselzeitliche Feld, ebenso fallen Werte von eindeutig saalezeitlichen Proben in das erwartete weichselzeitliche Feld.

Diese widersprüchlichen Ergebnisse können mehrere Ursachen haben:

1. Steinert hat trotz einer relativ großen Probenzahl nicht in allen Regionen des Landes gezählt. Es ist zu vermuten, daß die Streuung größer ist, als von ihm angenommen wurde. Darauf deuten auch die Ergebnisse von PIELES (1955) hin.

2. Teilweise haben die verschiedenen Autoren für ihre Zählungen unterschiedliche Korngrößenklassen gewählt: Z. B. FIEDLER die Fraktion $60-90 \mu \mathrm{m}$, STEINERT, HÖlting, PIELES und Weiss die Fraktion $100-200 \mu \mathrm{m}$, PIELES z. T. auch $60-100 \mu \mathrm{m}$ und 200-300 $\mu \mathrm{m}$, SCHLENKER sowie SCHRÖDER die Fraktion $63-355 \mu \mathrm{m}$. Dies hat zur Folge, daß sich Verschiebungen der Daten ergeben, weil bestimmte Schwerminerale - insbesondere Zirkon - bevorzugt mit kleineren Korngrößen auftreten, dagegen Minerale wie Granat und Hornblende sowie einige Aluminiumsilikate bevorzugt in den größeren Fraktionen zu finden sind.

3. Ein Vergleich der Zählungen aus den verschiedenen Publikationen erweckt den Eindruck einer z.T. 
Tab. 2: Schwermineralverteilung (Fraktion 60-250 $\mu \mathrm{m}$ ) in Geschiebemergeln Schleswig-Holsteins

(Entnahmestellen s. Tab. 1)

\begin{tabular}{|c|c|c|c|c|c|c|c|c|c|c|}
\hline & $\begin{array}{c}1 \\
\text { qsAG }\end{array}$ & $\begin{array}{c}2 \\
\text { qsJG }\end{array}$ & $\begin{array}{c}3 \\
\text { qsMG }\end{array}$ & $\begin{array}{l}4 \\
\text { qs }\end{array}$ & $\begin{array}{c}5 \\
\text { qsJG }\end{array}$ & $\begin{array}{c}6 \\
\text { qsAG }\end{array}$ & $\begin{array}{c}7 \\
\text { qsAG }\end{array}$ & $\begin{array}{c}8 \\
\mathrm{qs} A \mathrm{G}\end{array}$ & $\begin{array}{c}9 \\
\mathrm{qsJG}\end{array}$ & $\begin{array}{l}10 \\
\text { qe }\end{array}$ \\
\hline OPAK & 34.6 & 44.4 & 35.3 & 25.1 & 22.5 & 34.5 & 25.6 & 43.1 & 40.2 & 43.8 \\
\hline EPIDOT & 16.7 & 14.0 & 20.5 & 22.2 & 15.8 & 15.2 & 17.0 & 11.7 & 16.8 & 20.1 \\
\hline HORNBLENDE & 18.6 & 20.1 & 23.5 & 25.3 & 29.5 & 20.5 & 24.8 & 11.7 & 15.9 & 15.8 \\
\hline GRANAT & 14.7 & 9.1 & 6.8 & 7.5 & 12.3 & 15.2 & 17.2 & 10.7 & 10.6 & 6.5 \\
\hline TURMALIN & 3.9 & 1.9 & 3.4 & 4.3 & 4.4 & 3.8 & 5.9 & 5.0 & 3.7 & 3.1 \\
\hline ZIRKON & 4.5 & 1.7 & 3.2 & 2.7 & 2.3 & 2.4 & 1.2 & 8.9 & 3.9 & 4.6 \\
\hline DISTHEN & 1.8 & 1.4 & 1.6 & 1.1 & 1.3 & 1.5 & 1.0 & 0.4 & 1.5 & 1.9 \\
\hline STAUROLITH & 1.2 & 1.4 & 0.2 & 1.0 & 2.1 & 0.8 & 0.0 & 0.8 & 1.2 & 0.8 \\
\hline ANDALUSIT & 0.0 & 0.0 & 0.0 & 0.1 & 1.2 & 0.2 & 0.4 & 0.4 & 0.6 & 0.2 \\
\hline KLINOPYR. & 0.4 & 1.2 & 0.8 & 1.4 & 1.7 & 1.0 & 1.4 & 0.8 & 0.0 & 0.0 \\
\hline RUTIL & 0.8 & 0.2 & 0.8 & 1.0 & 0.6 & 1.8 & 0.8 & 1.7 & 1.5 & 1.5 \\
\hline TITANIT & 0.0 & 0.0 & 0.0 & 0.4 & 0.0 & 0.0 & 0.0 & 0.0 & 0.4 & 0.0 \\
\hline MONAZIT & 0.6 & 0.2 & 0.6 & 0.8 & 0.4 & 0.2 & 0.6 & 0.0 & 0.8 & 0.4 \\
\hline APATIT & 0.0 & 0.4 & 0.6 & 2.3 & 1.5 & 1.2 & 1.2 & 1.9 & 0.6 & 0.4 \\
\hline SEKUNDÄRE & 0.4 & 1.2 & 0.8 & 1.4 & 0.0 & 0.4 & 0.6 & 1.4 & 1.0 & 0.4 \\
\hline SONSTIGE & 0.8 & 0.0 & 0.4 & 0.8 & 0.6 & 0.2 & 1.0 & 0.4 & 0.2 & 0.0 \\
\hline ZOISIT & 1.0 & 0.6 & 0.8 & 1.0 & 1.7 & 1.2 & 0.8 & 0.2 & 0.8 & 0.6 \\
\hline BIOTIT & 0.0 & 2.3 & 0.6 & 1.9 & 1.9 & 0.0 & 0.8 & 1.0 & 0.4 & 0.0 \\
\hline SUMME & 100.0 & 100.1 & 99.9 & 100.3 & 99.8 & 100.1 & 100.3 & 100.1 & 100.1 & 100.1 \\
\hline $\begin{array}{c}11 \\
\text { qsJG }\end{array}$ & $\begin{array}{c}12 \\
\mathrm{qs} M G\end{array}$ & $\begin{array}{c}13 \\
\text { qsJG? }\end{array}$ & $\begin{array}{l}14 \\
\text { qw }\end{array}$ & $\begin{array}{c}15 \\
\text { qsJG }\end{array}$ & $\begin{array}{c}16 \\
\text { qsAG }\end{array}$ & $\begin{array}{l}17 \\
\text { qw }\end{array}$ & $\begin{array}{l}18 \\
\text { qe }\end{array}$ & $\begin{array}{l}19 \\
\text { qe }\end{array}$ & $\begin{array}{l}20 \\
q w\end{array}$ & $\begin{array}{c}21 \\
\mathrm{qs} M G\end{array}$ \\
\hline 38.7 & 36.1 & 31.2 & 30.7 & 46.0 & 32.5 & 31.6 & 42.3 & 42.0 & 36.8 & 26.4 \\
\hline 14.5 & 12.8 & 16.5 & 10.3 & 9.6 & 17.1 & 14.4 & 18.4 & 15.8 & 9.9 & 14.6 \\
\hline 18.5 & 21.5 & 21.3 & 29.7 & 15.9 & 22.0 & 24.6 & 15.7 & 15.2 & 25.1 & 27.6 \\
\hline 13.8 & 14.0 & 11.8 & 10.7 & 14.7 & 11.5 & 11.3 & 6.3 & 8.1 & 10.2 & 8.8 \\
\hline 2.1 & 3.1 & 3.3 & 2.9 & 2.6 & 4.6 & 3.1 & 2.6 & 1.4 & 4.2 & 2.7 \\
\hline 4.7 & 2.0 & 3.7 & 5.6 & 4.2 & 1.2 & 3.5 & 3.5 & 1.7 & 3.1 & 1.4 \\
\hline 1.3 & 1.3 & 1.2 & 2.2 & 1.2 & 1.8 & 1.4 & 3.4 & 10.3 & 1.8 & 2.1 \\
\hline 1.9 & 1.5 & 1.4 & 1.0 & 1.1 & 1.3 & 0.6 & 0.6 & 0.3 & 0.0 & 1.8 \\
\hline 0.6 & 2.0 & 0.6 & 0.5 & 0.2 & 0.0 & 0.6 & 0.2 & 0.0 & 0.0 & 0.1 \\
\hline 0.0 & 0.4 & 1.2 & 0.7 & 1.1 & 1.3 & 1.6 & 0.4 & 0.3 & 1.3 & 1.7 \\
\hline 1.0 & 0.2 & 1.0 & 1.0 & 1.1 & 1.7 & 1.6 & 1.3 & 1.8 & 1.6 & 0.6 \\
\hline 0.2 & 0.2 & 0.2 & 0.2 & 0.2 & 0.2 & 1.2 & 0.4 & 0.1 & 0.3 & 0.4 \\
\hline 0.4 & 0.5 & 0.2 & 0.8 & 0.2 & 0.6 & 0.4 & 0.2 & 0.6 & 0.3 & 0.1 \\
\hline 0.0 & 0.2 & 1.2 & 0.5 & 0.5 & 0.4 & 0.8 & 1.1 & 0.4 & 1.8 & 1.3 \\
\hline 0.4 & 1.6 & 1.0 & 0.5 & 0.4 & 1.3 & 1.0 & 1.5 & 1.0 & 1.6 & 2.6 \\
\hline 0.0 & 0.4 & 0.2 & 0.5 & 0.2 & 0.4 & 0.6 & 0.4 & 0.1 & 0.5 & 0.9 \\
\hline 1.3 & 0.5 & 1.0 & 1.0 & 0.5 & 0.2 & 0.4 & 0.2 & 0.6 & 1.0 & 1.6 \\
\hline 0.4 & 1.6 & 3.1 & 1.2 & 0.5 & 1.7 & 1.6 & 1.7 & 0.4 & 0.5 & 5.3 \\
\hline 99.8 & 99.9 & 100.1 & 100.0 & 100.2 & 99.8 & 100.3 & 100.2 & 100.1 & 100.0 & 100.0 \\
\hline
\end{tabular}


recht subjektiven Mineralansprache. Damit wären die Zähltabellen häufig fehlerhaft, eine Feststellung, die ähnlich auch schon ILLERS, RUDOLPH \& SEIM (1963: 890) trafen. Möglicherweise sind die deutlichen Unterschiede in den Prozentangaben für „stabile” Schwerminerale bei MEYER \& SCHLENKER (1979) gegenüber STEINERT (1952) nicht nur auf die gewählte unterschiedliche Korngrößenfraktion zurückzuführen, sondern beruhen auch auf Anspracheunterschieden.

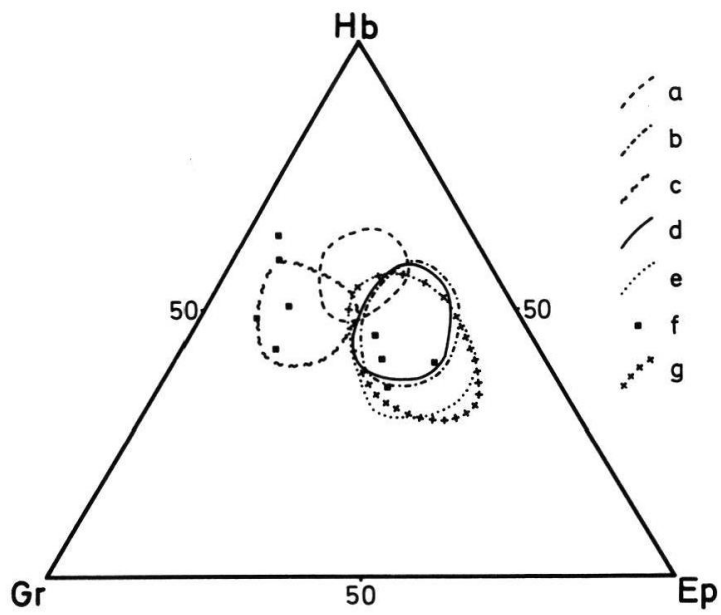

Abb. 2: Granat: Hornblende : Epidot + Zoisit aus Geschiebemergeln Schleswig-Holsteins; Symbole s. Abb. 1.

Abbildung 2 zeigt die Projektion der Daten im Dreiecksdiagramm Hornblende-Epidot-Granat. In Abb. 3 werden sie mit den Ergebnissen von SCHLENKER's Zählungen aus dem nördlichen Niedersachsen zusammen dargestellt. Aus diesen Abbildungen wird deutlich, daß sich Proben unterschiedlichen Alters in verschiedenen Teilflächen der Diagramme häufen. So ist das Feld mit weichselzeitlichen Proben eindeutig in Richtung zunehmender Hornblendeanteile verschoben. Ältere Geschiebemergel führen mehr Epidot und z. T. auch mehr Granat. Innerhalb der Gruppe der älteren Geschiebemergel haben die Proben des jüngeren saalezeitlichen roten Geschiebemergels (Warthe) und z. T. der roten Fazies des älteren saalezeitlichen Geschiebemergels (Drenthe) höhere Granatgehalte. Offenbar zeichnet sich hierin ein ähnliches Herkunftsgebiet ab. Beide Geschiebemergel stellen die von der jeweiligen Normalfazies abweichenden, viel ostbaltisches Material enthaltenden Ablagerungen aus der späten Phase der zugehörigen Vereisung dar.

Interessanterweise ist ein Teil der saalezeitlichen älteren Geschiebemergel in ,,roter” Fazies nicht durch höhere Granatgehalte gekennzeichnet, sondern fällt im Diagramm in den Bereich, in dem auch die Proben

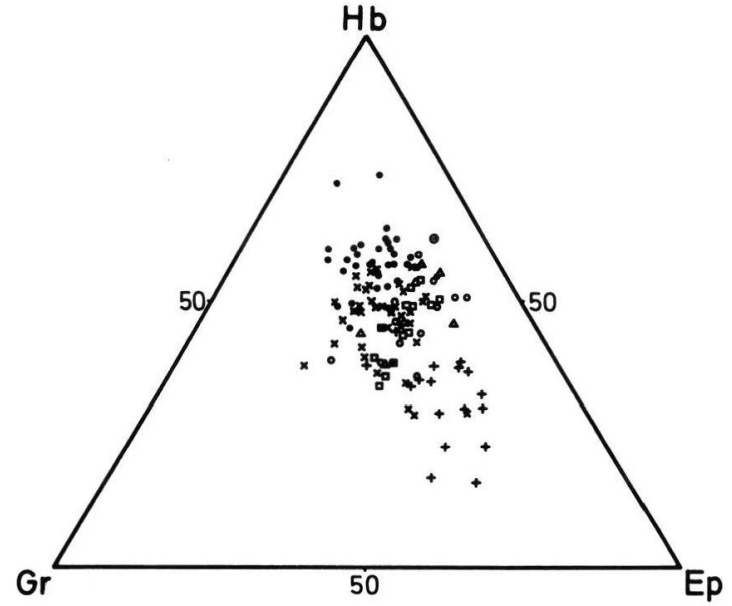

Abb. 3: Schwerpunkt-Streufelder im Diagramm Granat: Hornblende : Epidot + Zoisit

Proben aus Geschiebemergeln Nordwestdeutschlands.

Einzelne Proben liegen z. T. weit außerhalb der umrissenen Felder.

$a=$ weichselzeitlich $; b-f=$ saalezeitlich $(b=$ jüngerer $\mathrm{c}=$ roter jüngerer, $\mathrm{d}=$ mittlerer, $\mathrm{e}=$ älterer,

$\mathrm{f}=$ roter älterer Geschiebemergel); $\mathrm{g}=$ elsterzeitlich

der „Normalfazies” liegen. Möglicherweise deutet sich hierin eine Trennung dieses älteren ,roten” Geschiebemergels in zwei verschieden zusammengesetzte Teilfazies an. Die Angabe von FIEDLER (1939: $333 \mathrm{f}$ ), daß besonders weichselzeitliche Proben granatreich seien, ebenso die von HENNINGSEN (1987) angegebene Tendenz, daß der Granatgehalt in sandigen Glazialablagerungen von den älteren zu den jüngeren anstiege, müssen unter dem Aspekt, daß höhere Granatgehalte in verschiedenen älteren Geschiebemergeln vorkommen, in Frage gestellt werden. Vermutlich macht sich hier statistisch bemerkbar, daß in den betrachteten Regionen die jüngeren Ablagerungen häufiger als die älteren baltisch bis ostbaltisch geprägt sind. Regionale Unterschiede in der SM-Zusammensetzung sind ja seit langem bekannt (z. B. FIEDLER 1940: 214). FIEDLER (vgl. auch STEINERT 1952: 109) weist auch auf die auffällig hohen Granatgehalte der jüngsten Ablagerungen nördlich und südlich von Flensburg hin. Diese Tatsache gab offenbar WEISS (1958) die Möglichkeit, weichselzeitliches und älteres Material besser als allein mit Hilfe der „Hornblendezahl" zu trennen. Möglicherweise ist der Granatreichtum der äußersten weichselzeitlichen Ablagerungen bei Flensburg Folge eines „altbaltischen” Vorstoßes, wie er im Gebiet der dänischen Inseln bekannt ist, in Schleswig-Holstein bisher aber nicht nachgewiesen wurde.

In den Diagrammen ist eine klare Trennung der verschieden alten Moränen nicht möglich. Insbesondere 
scheitert der Versuch, die Moränen der verschiedenen saalezeitlichen Gletschervorstöße zu trennen, sowohl in ihren Normalausbildungen als auch in der ostbaltisch geprägten Fazies. Allenfalls könnte normaler Granatgehalt in einer roten Moräne auf den älteren saalezeitlichen Gletschervorstoß hindeuten.

Generell streuen die Werte aus den saalezeitlichen Proben stark, und es gibt Überschneidungen des saalezeitlichen mit dem weichselzeitlichen Feld. Tendenziell zeichnen sich die älteren von den mittleren saalezeitlichen Proben durch etwas niedrigere Hornblendewerte aus. Die Daten des jüngeren saalezeitlichen Geschiebemergels streuen dagegen sehr stark und sind nicht von den Daten der mittleren und älteren saalezeitlichen Geschiebemergel zu unterscheiden. Möglicherweise hat der jüngere saalezeitliche Gletschervorstoß die älteren Sedimente oft stark ausgeschürft und erneut umgelagert.

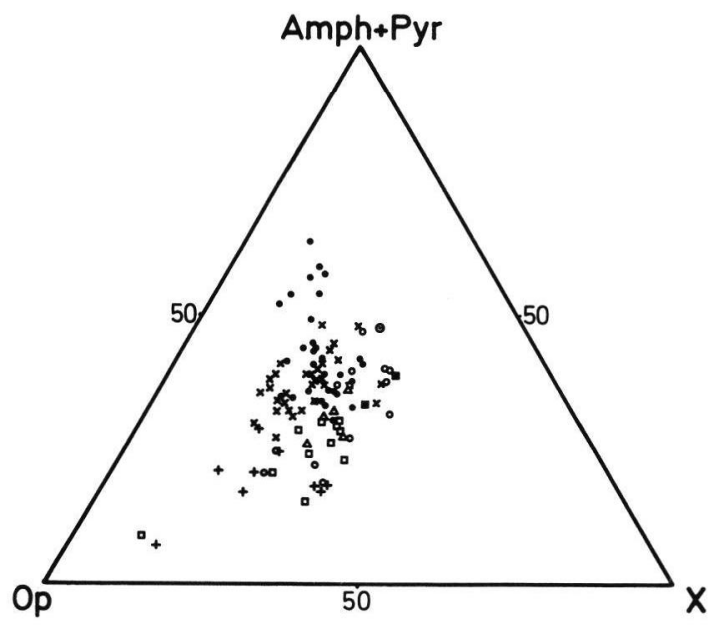

Abb. 4: Opake Schwerminerale: Amphibole + Pyroxene: stabile Schwerminerale $(\mathrm{X}=$ Epidot + Zoisit + Zirkon + Rutil + Disthen + Staurolith + Sillimanit + Andalusit + Turmalin).

Abb. 4 gibt einen weiteren Auswerteversuch wieder. Neben der verwitterungsempfindlichen Hornblendeund Augitgruppe und der Gruppe der stabilen Schwerminerale wird die Gruppe der opaken Schwerminerale genutzt. $\mathrm{Zu}$ der bereits aus $\mathrm{Abb} .1$ bis $3 \mathrm{ab}-$ lesbaren Tendenz der Verschiebung von Proben aus älteren Geschiebemergeln zum Bereich mit stabileren Mineralen erfolgt eine ausgeprägte Verlagerung in Richtung opake Minerale. Stärkere Überlappungen gibt es zwischen weichselzeitlichen und saalezeitlichen Proben insgesamt und zwischen saalezeitlichen und elsterzeitlichen Proben.

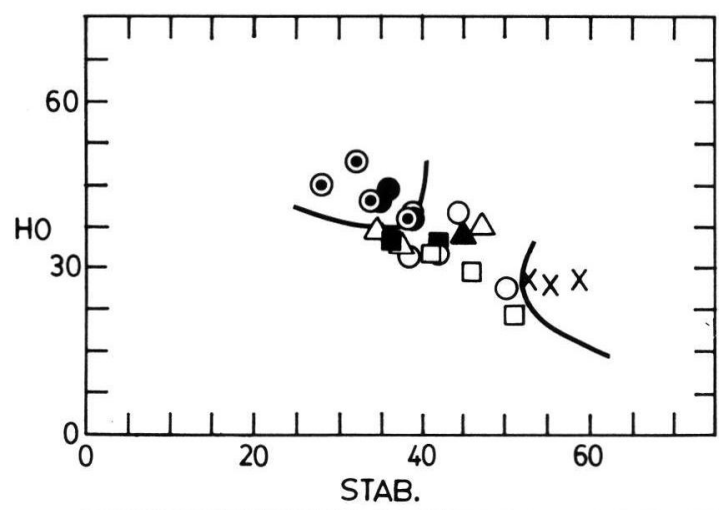

\begin{tabular}{|ll|}
\hline O HEILIGENHAFEN, qw & \\
qw & $\square$ qsAG, rot \\
$\Delta$ qs & $\square q S A G$ \\
O qSJG & $x$ qe \\
$\triangle$ qSMG & \\
\hline
\end{tabular}

Abb. 5: $\mathrm{HO}$ ( = Amphibole + Pyroxene) gegen stabile Schwerminerale (wie in Abb. 4). Werte aus Tab. 2 und Werte der oM-Proben aus Tab. 3.

Abb. 5 wertet nur die für diese Arbeit neu gezählten Proben im binären Diagramm Hornblende + Augite gegen „stabile” Schwerminerale aus. Die Zunahme stabiler Schwerminerale zu älteren Geschiebemergeln hin wird wieder deutlich.

\section{Schlußfolgerungen}

In die vergleichende Untersuchung wurden nur Analysen aus frischen, unverwitterten Moränen, den Geschiebemergeln, aufgenommen, ausgenommen in der Abb. 3 mit den Zählungen SCHLENKERs. Gerade seine Ergebnisse machen deutlich, daß man keine Zählungen aus verwittertem Material mit denen aus unverwittertem Material zusammenfassen sollte. So ermittelte SCHLENKER etwa in dem von Naßbleichung betroffenen Geschiebelehm der saalezeitlichen Moräne am Roten Kliff von Sylt Granatgehalte zwischen 0,7 und 3,7 Prozent. Im tiefsten, unverwitterten Teil der Moräne schnellt der Prozentsatz auf $11 \%$ empor.

Unter der Voraussetzung, daß einheitlich frisches Material untersucht wird, erscheint es mit Hilfe der Schwermineralanalyse möglich, relativ gut zwischen den jüngsten, den weichselzeitlichen Geschiebemergeln und den ältesten, den elsterzeitlichen, zu unterscheiden. Das veranschaulichen noch einmal in vereinfachender Darstellung die Abb. 6 und 7. Teilweise weitgehende Überschneidungen in der Zusammensetzung der Schwerminerale gibt es zwischen weichsel- 
HORNBLENDE

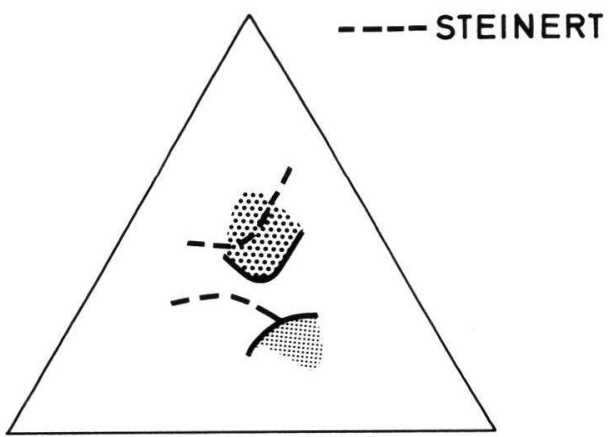

OPAK

STABILE GRANAT

HORNBLENDE

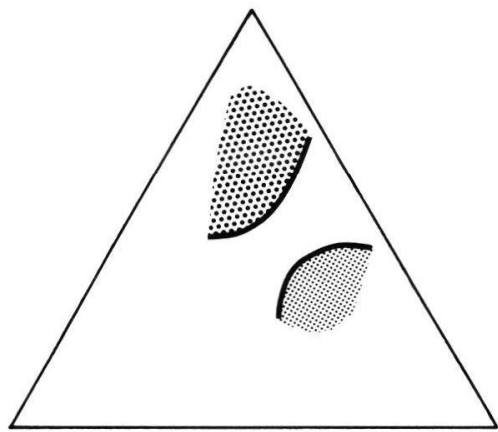

EPIDOT

Abb. 6 und 7: Grobes Raster $=$ weichselzeitliche Geschiebemergel, feines Raster = elsterzeitliche Geschiebemergel aus Schleswig-Holstein.

Tab. 3: Schwerminerale in Geschiebemergeln vom Heiligenhafener „Hohen Ufer” (Fraktion 63-355 $\mu \mathrm{m}$ ) Linke Spalte: Kornprozente; rechte Spalte: Flächenprozente

$\mathrm{oM}, \mathrm{mM}, \mathrm{uM}=$ oberer, mittlerer, unterer Geschiebemergel, oMr $=$ rote Schliere im oberen Geschiebemergel

\begin{tabular}{|c|c|c|c|c|c|c|c|c|c|}
\hline Probe & I & III & $\mathrm{V}$ & VI & VII & VIII & IX & $\mathrm{X}$ & XIII \\
\hline G.-MERGEL & $\mathrm{mM}$ & $\mathrm{uM}$ & $\mathrm{oM}$ & $\mathrm{oM}$ & $\mathrm{uM}$ & $\mathrm{uM}$ & $\mathrm{mM}$ & $\mathrm{oM}$ & $\mathrm{oMr}$ \\
\hline
\end{tabular}

OPAK

EPIDOT

HORNBLENDE

GRANAT

TURMALIN

ZIRKON

DISTHEN

STAUROLITH

SILLIMANIT

ANDALUSIT

PYROXEN

RUTIL

TITANIT

MONAZIT

APATIT

SONSTIGE

SEKUNDÄRE

BIOTIT
$27,0 / 19,0 \quad 28,1 / 26,6 \quad 28,9 / 27,8 \quad 26,6 / 25,3 \quad 34,8 / 32.3 \quad 28,6 / 28,1 \quad 32,4 / 24,0 \quad 23,9 / 21,5 \quad 19,7 / 22,0$ $16,8 / 7,5 \quad 19,1 / 20,2 \quad 16,4 / 14,6 \quad 15,3 / 19,0 \quad 14,6 / 16,8 \quad 17,2 / 13,3 \quad 16,0 / 11,4 \quad 16,3 / 14,1 \quad 18,3 / 18,1$ $27,7 / 47,3 \quad 27,9 / 26,2 \quad 26,5 / 32,0 \quad 28,6 / 27,0 \quad 27,6 / 27,4 \quad 29,7 / 24,4 \quad 25,8 / 28,3 \quad 32,4 / 28,9 \quad 37,8 / 35,9$

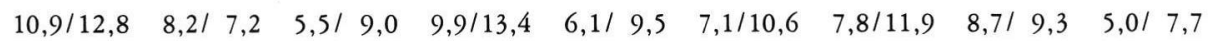
$2,2 / 5,1 \quad 3,2 / 3,4 \quad 4,3 / 3,2 \quad 2,2 / 4,5 \quad 0,9 / 4,2 \quad 1,5 / 5,2 \quad 2,8 / 5,0 \quad 1,6 / 2,6 \quad 2,4 / 3,5$

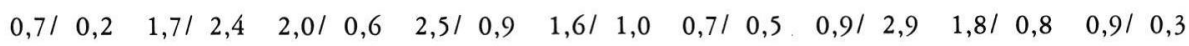
$\begin{array}{lllllllllllllllll}0,7 / & 0,5 & 0,2 / & 1,6 & 1,4 / 2,1 & 1,0 / & 0,6 & 1,4 / & 1,8 & 1,3 / 1,1 & 1,6 / 3,0 & 1,3 / 3,4 & 2,2 / & 2,0\end{array}$ $\begin{array}{lllllllllllllllllllll}1,1 / & 2,0 & 1,7 / & 0,5 & 2,0 / & 1,5 & 1,7 / 2,9 & 0,9 / & 0,7 & 0,2 / & 0,0 & 0,5 / & 1,9 & 1,3 / & 0,4 & 0,2 / & 0,0\end{array}$ $\begin{array}{llllllllllllllllllllllllll}0,0 / & 0,3 & 0,0 / & 0,0 & 0,0 / & 0,0 & 0,3 / & 0,0 & 0,5 / & 0,0 & 0,0 / & 0,0 & 0,0 / 0,0 & 0,2 / & 0,4 & 0,0 / & 0,0\end{array}$ $\begin{array}{lllllllllllllllll}0,4 / & 0,5 & 0,6 / & 0,3 & 0,6 / & 1,3 & 1,5 / & 0,3 & 0,5 / & 0,4 & 0,7 / 3,2 & 0,2 / & 0,0 & 0,7 / & 0,9 & 0,2 / & 1,0\end{array}$ $\begin{array}{llllllllllllllllll}1,1 / & 0,3 & 0,9 / & 1,3 & 1,0 / & 1,0 & 2,0 / & 0,7 & 1,1 / & 2,3 & 2,4 / 3,9 & 1,1 / & 1,6 & 1,6 / 2 / 5 & 2,2 / & 1,3\end{array}$ $\begin{array}{lllllllllllllllllll}0,2 / & 1,0 & 0,6 / & 0,9 & 0,4 / 2,0 & 0,3 / & 1,0 & 1,6 / & 0,4 & 0,7 / & 1,2 & 1,4 / & 3,4 & 1,6 / & 1,1 & 0,9 / & 0,0\end{array}$

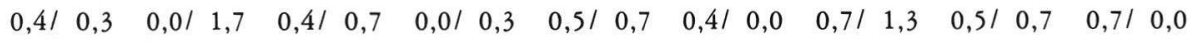
$\begin{array}{lllllllllllllllllll}0,0 / & 0,0 & 0,4 / & 0,0 & 0,6 / & 0,0 & 0,5 / & 0,5 & 0,2 / & 0,0 & 0,2 / & 0,9 & 0,2 / & 0,0 & 0,0 / & 0,0 & 0,4 / & 0,0\end{array}$ $\begin{array}{llllllllllllllllll}1,7 / & 1,4 & 1,5 / & 5,1 & 1,6 / & 2,5 & 1,7 / & 2,4 & 2,2 / & 2,0 & 2,4 / & 2,9 & 3,7 / & 4,8 & 3,1 / & 6,2 & 3,7 / & 3,7\end{array}$


$\begin{array}{llllllllllllllllll}4,8 / & 0,0 & 4,3 / & 1,3 & 7,4 / & 0,2 & 5,2 / & 0,0 & 4,9 / & 0,6 & 4,7 / & 2,4 & 3,7 / & 1,3 & 3,1 / & 7,1 & 4,6 / & 3,1\end{array}$




zeitlichen und saalezeitlichen Moränen einerseits und saalezeitlichen und elsterzeitlichen andererseits. Innerhalb der saalezeitlichen Geschiebemergel erscheint es nur möglich, die ostbaltisch geprägte Geschiebemergelfazies der jüngeren saalezeitlichen Vergletscherung (qsJG, Warthe) und teilweise der älteren saalezeitlichen Vergletscherung (qsAG, Drenthe), also viele der „roten Moränen” aufgrund ihres starken Granatgehaltes von den übrigen saalezeitlichen Moränen abzutrennen. Bei starker Granatführung sind die beiden „roten Moränen” nicht voneinander zu unterscheiden. Normale Granatführung in einer saalezeitlichen „roten Moräne” scheint auf den älteren Gletschervorstoß hinzuweisen.

Die beiden älteren Geschiebemergel vom Heiligenhafener "Hohen Ufer”, deren problematische zeitliche Zuordnung Anlaß für die vorliegende Untersuchung war, sollten nach den SM-Zählungen am ehesten weichselzeitliches Alter haben. Jedoch reicht unseres Erachtens für eine sichere Aussage die Analysenzahl in der Region Ostholsteins bisher bei weitem nicht aus.

Nicht nur für Schleswig-Holstein dürfte die Feststellung gelten, daß die Schwermineralanalyse - wie ähnlich auch die anderen bisher bei lithostratigraphischen Arbeiten eingesetzten petrographischen Methoden - ganz offensichtlich nur eine bestimmte Sedimentfazies kennzeichnet, im hier betrachteten Umfang also eine bestimmte Geschiebemergelfazies. Da das Inlandeis der verschiedenen Eiszeiten oder größeren Gletschervorstöße vom Ursprungsgebiet bis zum Abschmelzgebiet offenbar wiederholt denselben oder zumindest einen ähnlichen Weg nahm, wurden wiederholt dieselben Gesteine aufgenommen, was im Abschmelzgebiet wiederholt zur Ablagerung faziell ähnlicher Geschiebemergel (Moränen) führte (STEPHAN 1987: 29). Eine stratigraphische Aussage mit Hilfe petrographischer Daten wird damit prinzipiell eingeschränkt.

Speziell die Schwermineralanalyse weist, wie bereits erwähnt, abgesehen von der Subjektivität der Zählungen auch durch mangelnde Standardisierung in der Methodik Einschränkungen auf. So können beispielsweise Korngrößeneffekte bei den bisher insgesamt durchgeführten Kornzählungen nicht ausgeschlossen werden. Da bei Kornzählungen feinkornreiche Proben u.a. höhere Zirkongehalte erbringen als grobkornreiche, können erstere bei dem relativen Gewicht des Zirkons unter den durchsichtigen Schwermineralen insgesamt einen höheren Anteil an stabilen Mineralen besitzen. Sie verschieben sich damit im entsprechenden Auswertungsdiagramm zu den als älter eingestuften Probenserien. Beim Einbeziehen von ausgeprägt feinkornreichen Proben in die Auswertung brächte die Auszählung von Flächenprozenten eine
Verbesserung der Vergleichbarkeit. Diese Möglichkeit versagt aber, wenn besonders grobkornreiche Proben vorkommen. Einen Ausweg ergäbe wahrscheinlich nur die Wahl enger Korngrößenfraktionen, von denen evtl. zwei oder mehrere nebeneinander gezählt und getrennt ausgewertet werden könnten.

Fazielle Verschiebungen von Region zu Region innerhalb gleichalter Ablagerungen machen Korrelationsversuche noch schwieriger. Es bleibt wie bei den anderen petrographischen Methoden nur der Versuch, im regionalen und dann vielleicht auch recht engen regionalen Rahmen petrographische Charakteristika verschieden alter Geschiebemergel zu erarbeiten, um schrittweise eine zeitliche Einhängung benachbarter Räume zu erreichen.

Die Schwermineralanalyse erfordert dabei einen relativ hohen Arbeitsaufwand und bleibt trotzdem in der Aussageschärfe hinter den Ergebnissen anderer petrographischer Methoden wie z. B. der Feinkiesanalyse zurück. Es erscheint daher nicht sinnvoll, die Schwermineralanalytik in größerem Umfange für Korrelationsversuche einzusetzen. Eine mögliche Ausnahme wäre die Untergliederung von Bohrprofilen bei nur geringem Materialanfall. Wegen der nur kleinen benötigten Menge an Ausgangsmaterial könnte sie in diesen Fällen - wie auch die chemische Analyse (CHRISTENSEN 1979) - von Nutzen sein.

\section{Schriftenverzeichnis}

BiermanN, M. (1981): Sedimentologie und Geochemie der Weichsel-Moräne von Timmerhorn und quartärgeologische Kartierung auf dem Blatt 6450 Garstedterfeld (Maßstab 1 : 5000). - Dipl. Arbeit, Fachber. Geowiss., Univ. Hamburg: 127 S., Anhang mit 16 Tabellen und 10 Karten; Hamburg. - [Unveröff.]

BOENIGK, W. (1983): Schwermineralanalyse. - 152 S.; Stuttgart (Enke).

Christensen, S. (1979): Geochemische Untersuchungsmethoden zur stratigraphischen Einstufung glazigener Sedimente. - Verh. naturwiss. Ver. Hamburg (NF), 23: 39-46; Hamburg.

DUPHORN, K. (1983): Quartärgeologische Gesamtinterpretation Gorleben. Abschlußbericht für die PTB: 265 S., $41 \mathrm{Abb} ., 48$ Karten und Profile; Braunschweig (Archiv PTB). - [Unveröff.].

FiedLER, A. (1939): Ergebnisse der Schwermineralanalyse von Geschiebemergeln im mittleren und westlichen Norddeutschland. - Z. ang. Min., 1, 4: 313-352; Berlin.

- (1940): Ergebnisse der Schwermineralanalyse aus Holland, Dänemark, Oberschlesien und Norddeutschland, nebst einem Beitrag zur Frage nach dem absoluten Schwermineralgehalt der nordwestdeutschen Geschiebemergel. -Z. ang. Min. 2, 2: 199-218; Berlin. 
HENNINGSEN, D. (1987): Schwermineral-Kennzahlen von Quartär-Sedimenten Nordwestdeutschland. - Z. dt. geol. Ges., 138: 45-52; Hannover.

HÖFLE, H.-C. (1979): Klassifikation von Grundmoränen in Niedersachsen. - Verh. naturwiss. Ver. Hamburg (NF), 23: 81-92; Hamburg.

- \& SCHLENKER, B. (1979): Das Pleistozänprofil der Kreidegrube Hemmor bei Stade (Elbe-Weser-Dreieck). - Geol. Jb., A49: 3-25; Hannover.

HöltiNG, B. (1957): Schwermineralanalytische Untersuchungen in Riß- und Würmablagerungen im Gebiet der Kreise Segeberg, Plön und Rendsburg. - Heimatkdl. Jb. Kreis Segeberg, 1957: 155-176; Bad Segeberg.

Illers, K., RudolPh, H. \& Seim, R. (1963): Korngrößenund Schwermineralanalyse der Geschiebemergel der Stoltera bei Warnemünde. - Geologie, 12, 8: 889-916; Berlin.

LEINZ, V. (1933): Ein Versuch, Geschiebemergel nach dem Schwermineralgehalt stratigraphisch zu gliedern. $-\mathrm{Z}$. Geschiebeforsch., 9: 156-168; Leipzig.

MeYeR, K.-D. \& SCHLENKer, B. (1979): Abschlußbericht zum Projekt „Plio-Pleistozän Nordwestdeutschlands” (DFG-Projekt Me 433/2-4). - 39 S. und Anhang mit Abb. und Tab.; Hannover (Archiv NLfB). [Unveröff.].

PIELES, N. (1955): Diluvialgeologische Untersuchungen im Gebiet des Möllner Sanders. — Diss. Univ. Kiel: 84 S.; Kiel.
Pratje, O. \& RichteR, W. (1938): Stratigraphie und Schwermineralanalyse der beiden Geschiebemergel und des Dirschheimer Sandes an der Westküste des Samlandes Jb. preuß. geol. L.-Anst., 58: 666-676; Berlin.

RAISTRICK, A. (1929): The petrology of some Yorkshire Boulder Clays. - Geol. Magazine, 66, 8: 337-344; London.

STEINERT, H. (1952): Stratigraphie und Schwermineralprovinzen im Diluvium Schleswig-Holsteins und Norddeutschland. - Meyniana, 1: 107-111; Kiel.

STEPHAN, H.-J. (1985): Exkursionsführer Heiligenhafener „Hohes Ufer”. - Der Geschiebesammler, 18, 3: 83-99; Hamburg.

- (1987): Moraine stratigraphy in Schleswig-Holstein and adjacent areas; in J. J. M. Van der Meer (ed.): Tills and Glaciotectonics: 23-30; Rotterdam/Brookfield (A. A. Balkema).

WEISS, E. N. (1958): Bau und Entstehung der Sander vor der Grenze der Würmvereisung im Norden SchleswigHolsteins. - Meyniana, 7: 5-60, Tabellenanhang, Karten I-V; Kiel.

Manuskript eingegangen am 11. 10. 1988, Nachträge November 1988. 